\title{
THE INFLUENCE OF DEFORMATION AND CHEMICAL COMPOSITION ON ELEMENTARY FREE VOLUMES IN GLASSY POLYMERS
}

\author{
V.P. Shantarovich ${ }^{a}$, YU.A. Novikov ${ }^{b}$, Z.K. SuPtel ${ }^{a}$, E.F. OleiniK ${ }^{a}$ \\ AND M.C. BOYCE ${ }^{c}$
}

${ }^{a}$ Semenov Institute of Chemical Physics, Russian Academy of Sciences 4 Kosygina str., 117334, Moscow, Russia

${ }^{b}$ Institute of General Physics, Russian Academy of Sciences 38 Vavilov str., 117942, Moscow, Russia

'Department of Mechanical Engineering, Massachusetts Institute of Technology Cambridge, Massachusetts, USA

\begin{abstract}
A relation between positronium annihilation characteristics and concentration of elementary free volumes is considered for the case of positronium formation before its localization. We estimated the diffusion coefficient of nonlocalized positronium $\left(\approx 10^{-4} \mathrm{~cm}^{2} / \mathrm{s}\right)$. The positron annihilation lifetime studies of uniaxial compression and annealing of some glassy polymers revealed variations of the shape of elementary free volumes size-distributions.
\end{abstract}

PACS numbers: $78.70 . \mathrm{Bj}$

\section{Introduction}

The positron annihilation lifetime (PAL) measurements are very promising for the studies of elementary free volumes (EFV) in polymers, and many interesting correlations are found (see [1] and references therein). However, some problems of interpretation remain unsolved. Thus, the mode of origin of positronium (Ps) in $\mathrm{EFV}$ is uncertain, and, correspondingly, inconclusive are the kinetic equations, which are to be solved in order to find a dependence of long-lived component intensity on EFV concentration. We have some experience in this field [1-5] and give arguments in favour of $\mathrm{Ps}$ formation before its trapping. We discuss also the results on PATFIT and CONTIN processing of PAL spectra for plastically deformed and annealed copolyether Vectra, polycarbonate (PC), and poly(methyl methacrylate) PMMA. 


\section{Two models of Ps trapping}

In contrast to the assumption of Ps formation after the concurrent localization of $\mathrm{e}^{+}$and $\mathrm{e}^{-}$[6], we assumed [1] the existence of nonlocalized Ps and Ps trapping by EFV. In this case, the long-lived component intensity $I_{3}\left(I_{4}\right)$ is dependent only on the Ps trapping rate and annihilation rate in the trap, but not on the $\mathrm{e}^{+}$trapping rate. Therefore, variations of the components $I_{2}\left(\mathrm{e}^{+}\right)$and $I_{3}(\mathrm{Ps})$ are independent. To support our model, we give some examples of such independent variations in ethylene-propylene copolymers.

The nonlocalized positronium trapping rate is dependent particularly on Ps mobility and determines the dependence of $I_{3}\left(I_{4}\right)$ on EFV concentration $\left(\approx 10^{19} \mathrm{~cm}^{-3}\right.$ ). The mobility (diffusion coefficient of nonlocalized Ps) was found in experiments with porous poly(phenylene oxide): $D_{\mathrm{Ps}} \approx 1 \times 10^{-4} \mathrm{~cm}^{2} / \mathrm{s}$.

\section{Plastic deformation of glassy polymers}

We investigated the dependence of EFV size and concentration on the uniaxial plastic compression of polycarbonate (PC, $T_{\mathrm{g}}=145^{\circ} \mathrm{C}$ ), PMMA, $T_{\mathrm{g}}=120^{\circ} \mathrm{C}$ and its copolymers and also liquid-crystalline copolyether. Vectra $\left(T_{\mathrm{g}}=403^{\circ} \mathrm{C}\right)$. The residual deformation up to $\varepsilon_{\text {res }} \approx 40 \%$ produced the opposite relative variations of the number of EFV, calculated from PAL data: $-6 \%$ for PC and $+60 \%$ for the copolyether. The positive result was also obtained for PMMA [7].

At the beginning, we compared PAL characteristics of nondeformed copolymers [MMA(OMA $\left.)_{x}, x=0-20 \%\right]$ and their temperature induced relaxation after compression $\left(\varepsilon_{\text {res }}=20 \%\right)$. The intensity $\left(I_{3}\right)$ was found to be essentially dependent on the value of a high-temperature component $\varepsilon_{2}$ of relaxation of residual deformation and, consequently, on transformations of EFV, which were due to the segmental mobility. Furthermore, on deformation of $\mathrm{PC}$ and subsequent annealing of the sample, we were the first to observe (using the standard CONTIN procedure described by Gregory and Jean) a transition from bimodal size-distribution $f(R)$ of EFV to unimodal one. American positron annihilation group from MIT had measured bimodal $f(R)$ for deformed PMMA. PC result is seen in Fig. 1, where curve 1 corresponds to the original PC, curve 2 to the deformed sample, and curve 3 to the both deformed and original samples after annealing. It is remarkable that complete relaxation of the EFV size-distributions $f(R)$ comes after short annealing at temperatures below $T_{\mathrm{g}}\left(126^{\circ} \mathrm{C}\right)$, when some residual deformation still remains. This result, in combination with the first one, demonstrates a limited segmental mobility at $T \leq T_{\mathrm{g}}$ and also existence of a certain "free-volume-component" of plastic shear transformations.

\section{The influence of chemical composition}

The transformations of $f(R)$ similar to that for PC deformation (Fig. 1) were induced in PMMA by a small amount (1.3 wt\%) of incompatible low-molecular-mass plasticizer: diethyl siloxane oligomer (not illustrated here because of the lack of space). The main peak of the $f(R)$ distribution was in the same position $(0.27 \mathrm{~nm})$, as that for original PMMA (Fig. 2, curve 1). The scale of the effect 


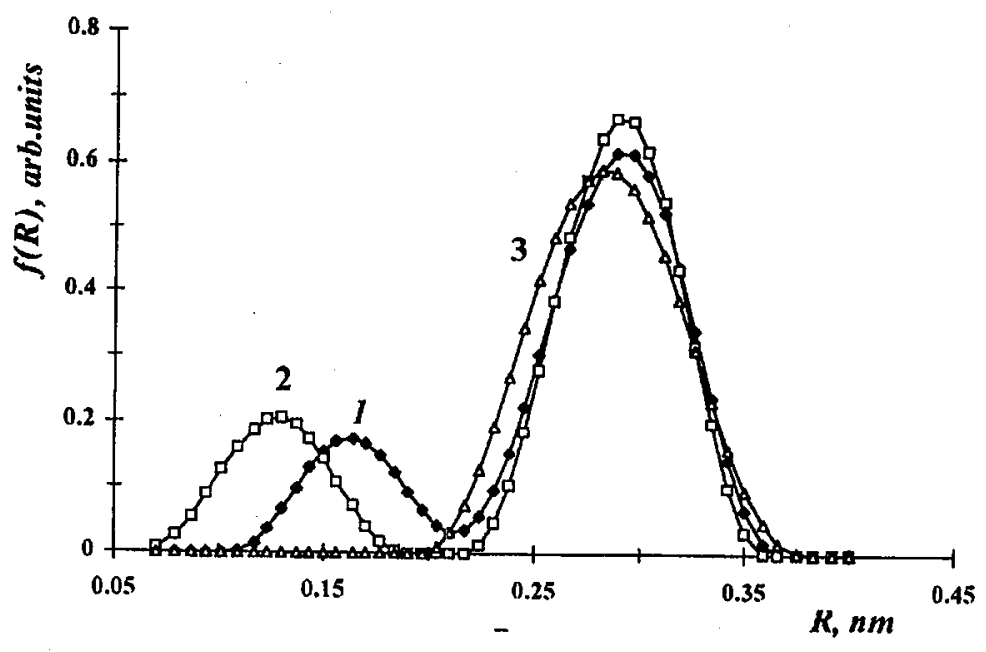

Fig. 1. Probability density function $f(R)$ of positronium annihilation in EFV of radius $R$ for polycarbonate (PC) with $T_{\mathrm{g}}=145^{\circ} \mathrm{C}$ : (1) original sample; (2) uniaxial compression with residual deformation $40 \%$; (3) after annealing at $T_{\mathbf{a n}}=126^{\circ} \mathrm{C}$.

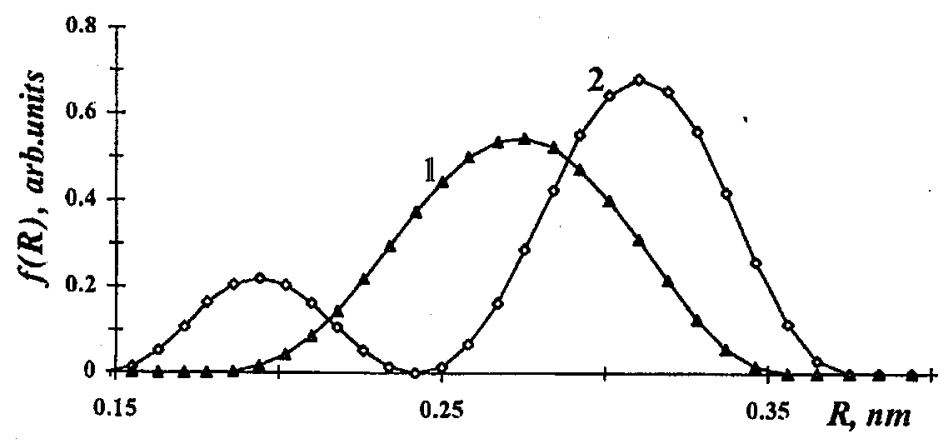

Fig. 2. Transition from the unimodal distribution $f(R)$ for PMMA (1) to bimodal one for the copolymer: MMA (80\%)-OMA (20\%) (2).

was much larger than it was expected from the amount of the admixture and demonstrates, probably, the formation of two phases with different EFV.

The effect of copolymerization of methyl methacrylate $80 \%$ and octhyl methacrylate 20\% (MMA-OMA) was slightly different (Fig. 2, curve 2). The main peak of the $f(R)$ distribution moved to larger EFV, thus illustrating the transformation of the whole matrix (compared to pure PMMA).

\section{Bimodal size-distribution of EFV}

The bimodal character of the probability density function of positronium lifetimes obtained using the CONTIN program may be interpreted (as we have done here) as a result of bimodal size-distribution of EFV. However, an alternative explanation can be found in the suggestion [8] that the distribution is actually 


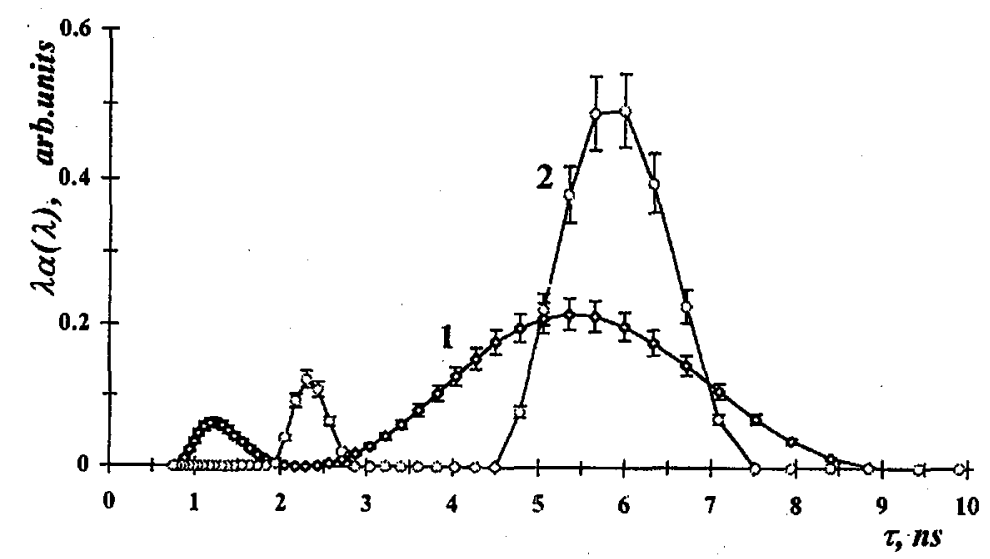

Fig. 3. Effect of atmospheric oxygen on probability density function $\lambda \alpha(\lambda)$ of Ps annihilation with a lifetime $r=\lambda^{-1}$ for the fluorine-containing polymer AF-1600: in air (1), in nitrogen atmosphere (2).

unimodal, but there are two different long-lived states of Ps: ortho-Ps and quasi-Ps, the latter annihilates mostly on the EFV walls and, therefore, is not sensitive to the pore content. The last assumption disagrees with quenching of the both positronium states by atmospheric oxygen in many polymer glasses, AF-1600 in particular (Fig. 3). Therefore, the conclusion on the bimodal size-distribution of EFV in some standard polymers glasses, such as modified PMMA and PC seems reasonable, too.

\section{Acknowledgments}

This work was supported by the Russian Foundation for Basic Research, projects no. 96-03-32468, 97-03-32762, 98-03-32859 and by the grant CRDF RE-2-205.

\section{References}

[1] V.P. Shantarovich, J. Radioanalytical Nucl. Chem. Articles 210, 357 (1996).

[2] V.P. Shantarovich, V.I. Goldanskii, Hyperfine Interact., in press, 1998.

[3] V.P. Shantarovich, Z.K. Azamatova, Yu.A. Novikov, Yu.P. Yampolskii, Macromolecules 31, 3963 (1998).

[4] V.P. Shantarovich, V.V. Gustov, E.F. Oleinik, I.B. Kevdina, O.B. Salamatina, Z.K. Azamatova, Vysokomolek. Soedineniya (Russ. Macromolecules) 40, No. 12 (1998).

[5] X.S. Li, M.C. Boyce, J. Polym. Sci. B, Polym. Phys. 31, 869 (1993).

[6] G. Dlubek, A.P. Clarke, H.M. Fretwell, S.B. Dugdale, M.A. Alam, Phys. Status Solidi A 157, 35 (1996).

[7] O.A. Hasan, M.C. Boyce, X.S. Li, S. Berko, J. Polym. Sci. B, Polym. Phys. 31, 185 (1993).

[8] G. Consolaty, F. Quasso, Appl. Phys. 50, 43 (1991). 